\title{
ANÁLISE DOS NÍVEIS DE METAIS EM ÁGUA SUBTERRÂNEA COLETADA À MONTANTE E JUSANTE DO ATERRO SANITÁRIO DE RIBEIRÃO PRETO, BRASIL
}

\author{
ANALYSIS OF METALS CONTENTS IN DOWSTREAM AND UPSTREAM SANITARY LANDFILL \\ GROUNDWATER COLLECTED SAMPLES, RIBEIRÃO PRETO, BRAZIL
}

\author{
Kamila de Almeida Piai ${ }^{1}$; Pricilla Costa Ferreira ${ }^{1}$; \\ Tânia Maria Beltramini Trevilato e Susana Inés Segura-Muñoz ${ }^{3}$
}

\begin{abstract}
Recebido em:09/12/2005, aceito em:15/09/2006
RESUMO Em Ribeirão Preto, há em funcionamento desde 1989, um aterro sanitário (AS) para resíduos domiciliares e um sistema de tratamento de resíduos de serviços de saúde (Sistema de Microondas). O objetivo deste estudo foi avaliar os níveis de metais em amostras de água subterrânea do lençol freático coletadas dos poços à montante e à jusante do Aterro Sanitário de Ribeirão Preto (ASRP). Foi avaliada a presença de $\mathrm{Ag}^{+}, \mathrm{Al}^{3+}, \mathrm{Ba}^{2+}, \mathrm{Ca}^{2+}, \mathrm{Cd}^{2+}, \mathrm{Cr}^{\circ}, \mathrm{Cu}^{\circ}, \mathrm{Mg}^{2+}, \mathrm{Mn}^{\circ}$, $\mathrm{Na}^{+}, \mathrm{Pb}^{\circ}, \mathrm{Hg}^{\circ}, \mathrm{K}^{+}, \mathrm{Se}^{2-}$ e $\mathrm{Zn}^{2+}$ por Espectrofotometria de Absorção Atômica. Os poços à montante do ASRP apresentaram, alterações de $\mathrm{Al}^{3+}, \mathrm{Mn}^{\circ}, \mathrm{Pb}^{\circ}$ e $\mathrm{Se}^{2-}$ acima do estabelecido pela Portaria 518/2004 do Ministério da Saúde (MS), Brasil, enquanto que os poços à jusante do ASRP apresentaram valores de $\mathrm{Mn}^{\circ}$ e $\mathrm{Pb}^{\circ}$ acima do estabelecido pela mesma Portaria. Comparando tais resultados com os obtidos em estudo realizado no ano 2000, para avaliação do nível dos metais $\mathrm{Cd}^{2+}, \mathrm{Cr}^{\circ}$, $\mathrm{Cu}^{\circ}, \mathrm{Hg}^{\circ}, \mathrm{Mn}^{\circ}, \mathrm{Pb}^{\circ}$ e $\mathrm{Zn}^{2+}$ na área de influência do ASRP, observa-se valores acima do estabelecido pela Portaria 1.469/2000 do Ministério da Saúde para $\mathrm{Mn}^{\circ}$ e $\mathrm{Pb}^{\circ}$ no solo e chorume, não havendo, ainda, a contaminação da água subterrânea na época. Os resultados obtidos no presente estudo trazem a necessidade de um constante monitoramento das águas do lençol freático a fim de minimizar ou impedir qualquer impacto ambiental concernente à contaminação por metais no local.
\end{abstract}

Palavras-Chave: Água Subterrânea; Metal; Resíduos sólidos.

\begin{abstract}
In Ribeirão Preto (Sao Paulo State, Brazil), a municipal landfill site (MLS) for urban waste disposal has been operating since 1989. It is integrated with the Medical Waste Treatment Plant (Microware System). This study aimed to estimate the metal levels in groundwater samples collected in downstream and upstream groundwater artesian wells of Ribeirão Preto Sanitary Landfill. It was estimated the presence of $\mathrm{Ag}^{+}, \mathrm{Al}^{3+}, \mathrm{Ba}^{2+}, \mathrm{Ca}^{2+}, \mathrm{Cd}^{2+}, \mathrm{Cr}^{\circ}, \mathrm{Cu}^{\circ}, \mathrm{Mg}^{2+}, \mathrm{Mn}^{\circ}, \mathrm{Na}^{+}$, $\mathrm{Pb}^{\circ}, \mathrm{Hg}^{\circ}, \mathrm{K}^{+}, \mathrm{Se}^{2-}$ e $\mathrm{Zn}^{2+}$ through Espectrophotometric of Atomic Absorption. The points in upstream presented levels of $\mathrm{Al}^{3+}, \mathrm{Mn}^{\circ}, \mathrm{Pb}^{\circ}$ and $\mathrm{Se}^{2-}$ higher than the standard established by Regulation 518/2004 of the Ministry of Health, of Brazil, while in downstream points the values of $\mathrm{Mn}^{\circ}$ and $\mathrm{Pb}^{\circ}$ were higher than the allowed limits. A study carried out in 2000, which estimated the levels of $\mathrm{Cd}^{2+}, \mathrm{Cr}^{\circ}, \mathrm{Cu}^{\circ}, \mathrm{Hg}^{\circ}, \mathrm{Mn}^{\circ}, \mathrm{Pb}^{\circ}$ and $\mathrm{Zn}^{2+}$ in the MLS area demonstrated $\mathrm{Mn}^{\circ}$ and $\mathrm{Pb}^{\circ}$ levels above the limits allowed by oficial regulations, in soil and sewage. However, groundwater samples collected in 2000 were not contaminated. The results obtained in the present study showed the need to adopt measures to protect the groundwater at the Sanitary Landfill Site Area, considering the detected levels of some metals that can affected the quality of the groundwater.
\end{abstract}

Keywords: Groundwater, Metal, Residue.

\section{INTRODUÇÃO}

O Brasil é dotado de um grande número de bacias subterrâneas, dentre as quais se destaca o Aquífero Guarani, um dos reservatórios mais importantes do mundo na atualidade (AGENDA 21 BRASILEIRA, 2000).

Grandes cidades brasileiras são abastecidas, total ou parcialmente, por água subterrânea procedente desse aquífero. Ribeirão Preto é um bom exemplo disso, já que $100 \%$ do seu abastecimento é proveniente do Aquífero Guarani (ALVEZ, 1995; VIARTI, 1999; PHILIPPI JÚNIOR e ALVES, 2004).
Segundo a Associação Brasileira de Águas Subterrâneas, há cerca de 200.000 poços tubulares em exploração e são perfurados aproximadamente 1.000 poços por ano no país. Atualmente, de acordo com os registros do SIDAS/DAEE e DAERP, no município de Ribeirão Preto são destinados 95 poços ao abastecimento público. Estima-se hoje, um consumo de $350 \mathrm{I} / \mathrm{dia} / \mathrm{hab}$., incluindo perdas na rede, totalizando um volume explorado de cerca de $176.000 \mathrm{~m}^{3} /$ dia (SÃO PAULO, 2004).

\footnotetext{
${ }^{1}$ Curso de graduação da Escola de Enfermagem de Ribeirão Preto-EERP/USP (kamilapiai@yahoo.com.br) (sininha83@yahoo.com.br)

${ }^{2}$ Faculdade de Medicina de Ribeirão Preto, Universidade de São Paulo. Setor de Metais. Laboratório de Pediatria do Hospital das Clínicas. Faculdade de Medicina de Ribeirão Preto/USP ( tmbeltra@fmrp.usp.br)

${ }^{3}$ Departamento Materno-Infantil e Saúde Publica da Escola de Enfermagem de Ribeirão Preto; EERP/USP-Laboratório de Saúde Ambiental (susis@eerp.usp.br)
} 
Notando-se a importância da água o abastecimento público, bem como em todo território nacional, considera-se necessário medidas urgentes de controle, monitoramento e redução da carga de agrotóxicos, metais e outros contaminantes químicos em mananciais, sob pena de vir a ter sérios problemas de poluição. O aumento na quantidade e variedade dos resíduos sólidos gerados pelas atividades domésticas, sociais e industriais emerge como um dos problemas prioritários do município, considerando o impacto do seu gerenciamento nos corpos de água subterrânea (ALMEIDA e CARNEIRO, 2001).

No município de Ribeirão Preto, S.P., a partir de 1990 a disposição de resíduos sólidos passou a ser executada em um aterro sanitário construído de acordo com as normas técnicas para receber resíduos sólidos do tipo classe II e III (ABNT,1987). Após avaliação, foi selecionada uma área de $201.400 \mathrm{~m}^{2}$, dentro da qual o ASRP passou a ocupar $135.000 \mathrm{~m}^{2}$ (Módulo I), localizado sobre a formação basáltica da Serra Geral, a qual não é considerada geológicamente uma zona de recarga do aquífero. Na atualidade, o ASRP encontra-se funcionando numa fase de extensão de $75.000 \mathrm{~m}^{2}$ (Módulo II) (SEGURAMUÑOZ, 2002). subterrânea no município de Ribeirão Preto para

Considerando o risco de contaminação inerente à disposição dos resíduos sólidos, e a importância dos recursos hídricos para a manutenção da vida, o presente projeto teve como objetivo a monitoração dos níveis de metais em água do lençol freático, na área do ASRP, S.P, considerada de risco potencial para poluição ambiental.

\section{MATERIAL E MÉTODOS}

\section{Local de estudo}

O município de Ribeirão Preto está localizado na região Nordeste do Estado de São Paulo, e atualmente possui uma população de cerca de 551.312 habitantes e uma área de 651 $\mathrm{Km}^{2}$ (DATASUS, 2006). O AS desse município encontra-se nas margens da Rodovia Mário Donega, que oferece acesso às cidades de Dumont e Pradópolis, próximo ao Anel Viário, situado entre as coordenadas $21^{\circ} 10^{\prime}$ a $21^{\circ} 15^{\prime}$ de latitude Sul e $47^{\circ} 50^{\prime}$ a $47^{\circ} 51^{\prime}$ de longitude Oeste.

As amostras de água foram coletadas de seis poços de monitoramento, dois localizados à montante e quatro à jusante do ASRP (Fig.1), em fevereiro de 2005.

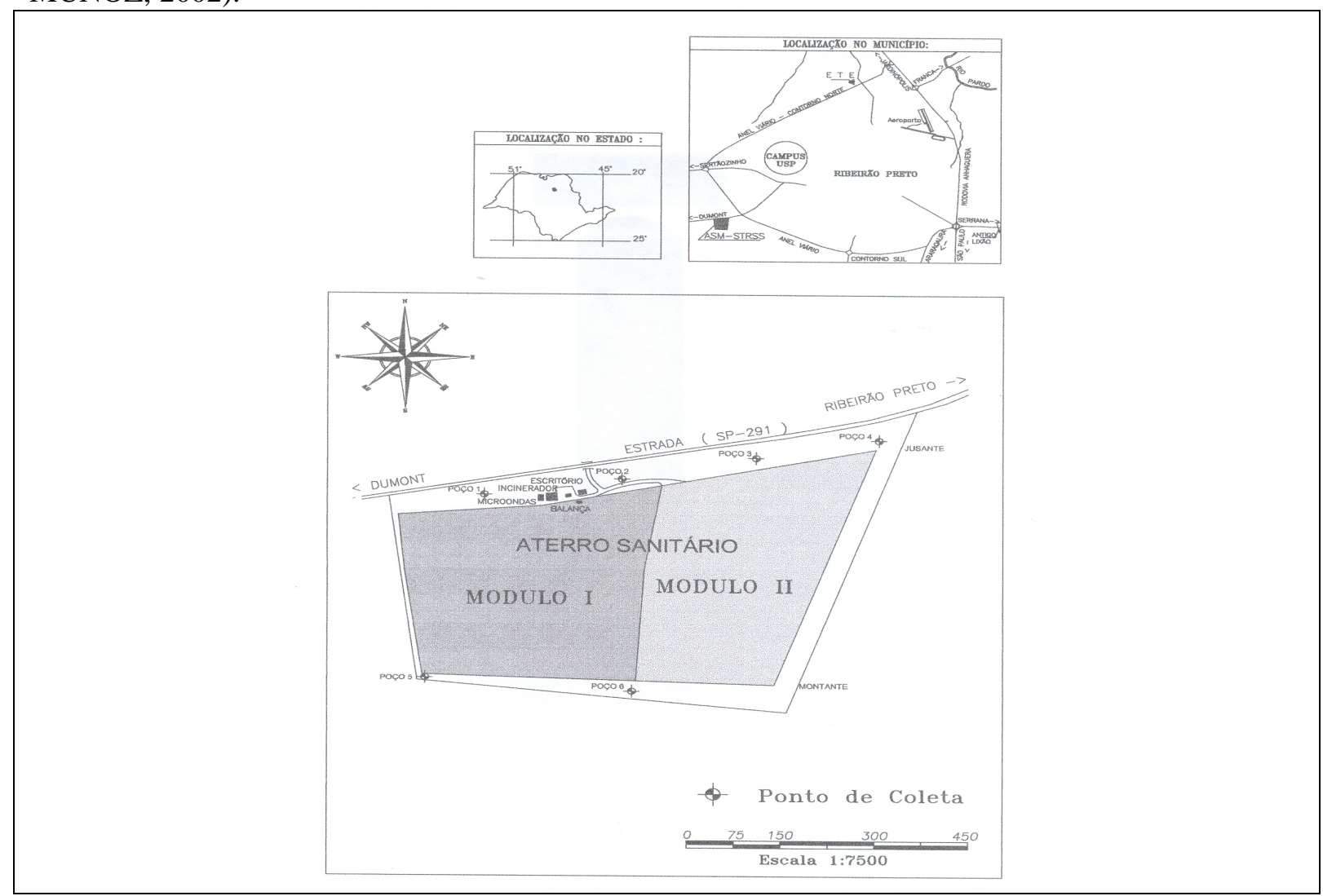

Figura 1. Representação gráfica dos pontos de coleta de água do lençol freático na área do Aterro Sanitário de Ribeirão Preto, SP.

Figure 1. Graphic representation of groundwater sampling points in Ribeirão Preto Sanitary Landfill, Brazil. 


\section{Coleta de amostras}

A coleta das amostras foi realizada em triplicata a fim de garantir a precisão dos dados obtidos e diminuir a probabilidade de discrepâncias nos resultados. Para a coleta foram utilizados tubos cônicos de polietileno de $50 \mathrm{~mL}$, providos de tampa, previamente submergidos em solução de ácido nítrico a $30 \%$ por 24 horas, para eliminação de metais interferentes (APHA, 1998) e posteriormente enxaguados com água Milli-Q no Setor de Metais do Laboratório de Pediatria do Hospital das Clínicas da Faculdade de Medicina de Ribeirão Preto (HCFMRP/USP).

\section{Preparação das amostras}

No momento de coleta foi realizada a medição do nível da água dos poços, que apresentaram uma média de $25 \mathrm{~m}$. A purga dos poços foi realizada 24 horas antes da coleta de amostras. As amostras coletadas foram centrifugadas antes da acidulação no Setor de Metais do Laboratório de Pediatria (HCFMRP/USP), contudo uma amostra de cada triplicata foi separada para análise de $\mathrm{pH}$. As outras duas amostras de cada triplicata foram fixadas adicionando $\mathrm{HNO}_{3}$ de alta pureza e mantidas $\mathrm{a}-18^{\circ} \mathrm{C}$ até o momento das análises.

\section{Análise da concentração hidrogênio iônica (pH)}

A leitura do $\mathrm{pH}$ foi realizada nas amostras separadas para tal fim, pelo método potenciométrico, utilizando $\mathrm{pH}$ modelo Q400/QUIMIS com eletrodo combinado de baixa força iônica (QA-338/BFI) no Setor de Metais do Laboratório de Pediatria do HCFMRP/USP, 4 horas após a realização da coleta.

\section{Método de Extração de Metais}

As amostras de água foram centrifugadas antes da acidulação, a 2.500 r.p.m durante 10 minutos em centrífuga modelo CT-5.000 (Cientec/Equipamentos para laboratório), o sobrenadante separado para posterior leitura dos metais (APHA, 1998).

\section{Leitura de Metais}

As dosagens de $\mathrm{Al}^{3+}, \mathrm{Ba}^{2+}, \mathrm{Cd}^{2+}, \mathrm{Cr}^{\circ}$ e $\mathrm{Mn}^{\circ}$ foram realizadas por Espectrofotometria de Absorção Atômica com Forno de Grafite (EAAFG/VARIAN-ZEEMAN modelo 640-Z). A dosagem de $\mathrm{Hg}^{\circ}$ foi realizada por Espectrofotometria de Absorção Atômica com Geração de Hidreto (EAA-VGA/VARIAN, modelo AA-200). As dosagens de $\mathrm{Ag}^{+}, \mathrm{Ca}^{2+}$, $\mathrm{Cu}^{\circ}, \mathrm{K}^{+}, \mathrm{Mg}^{2+}, \mathrm{Na}^{+}, \mathrm{Pb}^{\circ}, \mathrm{Se}^{2-}$ e $\mathrm{Zn}^{2+}$ foram realizadas por Espectrofotometria de Absorção Atômica de Chama (EAA-Chama/VARIAN, modelo AA-55) no Setor de Metais do Laboratório de Pediatria do HCFMRP/USP.

\section{Limites de detecção}

Os limites de detecção dos métodos utilizados para a análise dos metais são: $\mathrm{Cd}^{2+}$, $\mathrm{Hg}^{\circ}$ e $\mathrm{Pb}^{\circ} 0,0001 \mathrm{mg} / \mathrm{L} ; \mathrm{Al}^{3+}, \mathrm{Mn}^{\circ}$ e $\mathrm{Zn}^{2+}, 0,001$ $\mathrm{mg} / \mathrm{L} ; \mathrm{Cr}^{\circ}$ e $\mathrm{Ag}^{+} 0,002 \mathrm{mg} / \mathrm{L} ; \mathrm{Cu}^{\circ} 0,005 \mathrm{mg} / \mathrm{L}$; $\mathrm{Se}^{2-} 0,008 \mathrm{mg} / \mathrm{L}, \mathrm{Ba}^{2+} 0,02 \mathrm{mg} / \mathrm{L} ; \mathrm{Ca}^{2+}, \mathrm{K}^{+} \mathrm{e} \mathrm{Na}^{+}$ 1,0 mg/L e Mg 2,0 mg/L.

\section{Validação dos Métodos}

Foram utilizadas amostras certificadas de água potável do Instituto Quality Control Technologies Pty Ltd., Queensland, Austrália (PW-273, PW-278, PW-337 e PW-341).

\section{Análise dos dados}

Os dados foram analisados considerando os valores da Portaria 518/2004 do Ministério da Saúde (MS), que estabelece os procedimentos e responsabilidades relativas ao controle e vigilância da qualidade da água para consumo humano (BRASIL, 2004).

\section{RESULTADOS E DISCUSSÃO}

Considerando que aterros sanitários são locais propícios para contaminação ambiental por haver a disposição de uma ampla variedade de resíduos, torna-se indispensável à manutenção de poços de monitoramento de água subterrânea, controlando-se, dessa forma, a contaminação da mesma (REBOUÇAS; AMORE, 2002). As concentrações de metais das amostras coletadas nos poços do ASRP, são apresentadas na Tab. 1 .

Além dos níveis de metais, foi realizada a leitura do $\mathrm{pH}$ nas amostras coletadas dos poços à montante e à jusante do ASRP, detectou-se variação de 4,88 a 5,47 e 5,23 a 6,05 , respectivamente.

A partir dos resultados apresentados na tabela 1 , pode-se observar que $\mathrm{Cu}^{\circ}, \mathrm{Hg}^{\circ}$ e $\mathrm{K}^{+}$ encontraram-se abaixo do limite de detecção tanto nos poços à montante, quanto nos poços à jusante do ASRP, dessa forma inferiores ao nível estipulado pela Portaria 518/2004 do MS de 2,0 $\mathrm{mg} / \mathrm{L}$ para $\mathrm{Cu}^{\circ}, 0,001 \mathrm{mg} / \mathrm{L}$ para $\mathrm{Hg}^{\circ}$ e $875 \mathrm{mg} / \mathrm{L}$ para $\mathrm{K}^{+}$. Manganês e $\mathrm{Na}^{+}$apresentaram valores acima do limite de detecção apenas nos poços à jusante do ASRP, porém, ainda muito inferiores ao estipulado pela referida Portaria de $50 \mathrm{mg} / \mathrm{L}$ para $\mathrm{Mg}^{2+}$ e $200 \mathrm{mg} / \mathrm{L}$ para $\mathrm{Na}^{+}$. Bário e $\mathrm{Zn}^{2+}$ apresentaram valores mais elevados nos poços à jusante, porém, ainda muito inferiores ao nível estipulado pela referida Portaria, de $0,7 \mathrm{mg} / \mathrm{L}$ para $\mathrm{Ba}^{2+}$ e $5,0 \mathrm{mg} / \mathrm{L}$ para $\mathrm{Zn}^{2+}$. 
O cálcio apresentou níveis significativamente inferiores ao estipulado pela referida Portaria de $250 \mathrm{mg} / \mathrm{L}$. Segundo estudo realizado em águas subterrâneas do Aqüífero Guarani Serra-Geral no Paraná, foi constatado $\mathrm{pH}$ médio igual a 7,32, variando entre 6 e 9,5. Nesse estudo, também foi observado teor médio de $\mathrm{Ca}^{2+}$ de $9,78 \mathrm{mg} / \mathrm{L}$, sendo a calcita a grande controladora dos teores desse elemento em águas com $\mathrm{pH}$ alcalino (BITTENCOURT et al., 2003; ROSA FILHO et al., 2005). Outro estudo realizado com água subterrânea do Aqüífero Guarani no Paraná, na porção centro-norte, apresentou valor de $\mathrm{pH}$ inferior a 7,5 e teor de $\mathrm{Ca}^{2+}$ entre 0,8 e 50,3 $\mathrm{mg} / \mathrm{L}$. Portanto, nota-se que valores mais altos de $\mathrm{Ca}^{2+}$ favorecem $\mathrm{pH}$ mais alcalino (ROSA FILHO et al., 2005). Enquanto que para as amostras analisadas no presente estudo os valores de $\mathrm{pH}$ apresentaram-se abaixo de 6,05, correspondendo com os baixos níveis de $\mathrm{Ca}^{2+}$ encontrados.

Ao avaliar as concentrações de $\mathrm{Se}^{2-}$, observase que todas as amostras apresentaram valores abaixo do limite de detecção, ou seja, 0,008 $\mathrm{mg} / \mathrm{L}$, com exceção de uma amostra à montante, que apresentou valor de $0,015 \mathrm{mg} / \mathrm{L}$. O valor máximo permitido segundo a Portaria 518/2004 do MS para $\mathrm{Se}^{2-}$ é de $0,01 \mathrm{mg} / \mathrm{L}$. Esse metal pode ser encontrado em vidros e aços inoxidáveis. No organismo aumenta a incidência de cáries dentárias e possui potencial carcinogênico. Estudo realizado na região de Kelheim, sudoeste da Alemanha avaliou o teor de $\mathrm{Se}^{2-}$ presente em águas de lençol freático, no centro da cidade e em áreas adjacentes a uma fábrica de celulose e a uma fábrica produtora de ácido sulfúrico, onde eram depositados óxidos de ferro e piritas usadas na produção do ácido. Como consequiência da lixiviação, as amostras apresentaram altas concentrações de $\mathrm{Mn}^{\circ}$ e $\mathrm{Se}^{2-}$ entre elementos de menor concentração. A análise das amostras revelou ser o Se (VI) a espécie predominante, apresentando concentração variante entre $0,005 \mathrm{e}$ $0,328 \mathrm{mg} / \mathrm{L}$ (AZEVEDO e CHASIN, 2003).

Ao avaliar as concentrações de $\mathrm{Al}^{3+}$, observase que todos os poços apresentaram valores abaixo do estipulado pela Portaria 518/2004 do MS que é de $0,2 \mathrm{mg} / \mathrm{L}$, com exceção de uma amostra à montante, que apresentou valor de $0,272 \mathrm{mg} / \mathrm{L}$. A crescente aplicação do $\mathrm{Al}^{3+}$ na indústria aumentou nosso contato com o mesmo, como consequiência os resíduos gerados e dispostos em aterros a céu aberto e sanitários passam a propiciar maior potencial de contaminação desse metal no solo, chorume e conseqüentemente, na água subterrânea (PASCALICCHIO, 2002).

Em sistemas subterrâneos a solubilidade do $\mathrm{Al}^{3+}$ em equilíbrio com a fase sólida é altamente dependente do $\mathrm{pH}$, limites entre 4 a 6 geram hidrólise dos complexos contendo $\mathrm{Al}^{3+}$, deixando o metal livre (AZEVEDO e CHASIN, 2003).

Os achados no presente estudo referentes à concentração de $\mathrm{Al}^{3+}$ são relevantes, considerando que atualmente no contexto da Saúde Pública, infere-se que o aumento da concentração do $\mathrm{Al}^{3+}$ no organismo humano está relacionado com alterações neurológicas, evidenciando-se um índice elevado desse metal em pacientes portadores do Mal de Alzheimer (FERREIRA et al., 2004).

Ao avaliar as concentrações de $\mathrm{Mn}^{\circ}$, observa-se à montante uma média de $0,14 \mathrm{mg} / \mathrm{L}$, enquanto que à jusante do ASRP a média foi de $0,09 \mathrm{mg} / \mathrm{L}$. Cabe destacar que o estabelecido para o $\mathrm{Mn}^{\circ}$ é de $0,1 \mathrm{mg} / \mathrm{L}$ pela Portaria 518/2004 do MS. Considera-se que atualmente a fabricação de ligas metálicas e células secas sejam os principais usos do $\mathrm{Mn}^{\circ}$. Os valores encontrados podem representar a presença desses materiais, geralmente oriundos de processos desenvolvidos nas indústrias de pequeno porte dos setores que estão alocados no município, dentre os resíduos que são encaminhados ao ASRP. O transporte de $\mathrm{Mn}^{\circ}$ é favorecido, principalmente, pelas variações de $\mathrm{pH}$. Estudos demonstram que em meio ácido, ele circula na forma livre (AZEVEDO e CHASIN, 2003).

$\mathrm{O} \mathrm{Mn}^{\circ}$ é um mineral essencial, porém tóxico quando absorvido em excesso; os primeiros sintomas são debilidades musculares, tremores anormais, dor de cabeça, inclinação ao caminhar, salivação e reações psicóticas, quadro que pode desencadear sinais da Doença de Parkinson (SEGURA-MUÑOZ, 2003).

Quanto ao $\mathrm{Pb}^{\circ}$, em poços à montante apresentou média de $0,18 \mathrm{mg} / \mathrm{L}$, enquanto que à jusante do ASRP, apresentou média de 0,04 $\mathrm{mg} / \mathrm{L}$. O valor máximo permitido para $\mathrm{Pb}^{\circ}$ é de 0,01 mg/L segundo a Portaria 518/2004 do MS. Tais valores apontam a necessidade de avaliar a possibilidade de depósito de materiais como baterias, pigmento e outros produtos que apresentam esse metal em sua composição no ASRP (SEGURA-MUÑOZ, 2002). Observou-se em estudos realizados que em $\mathrm{pH}>5,4 \quad \mathrm{a}$ solubilidade total do $\mathrm{Pb}^{\circ}$ é, aproximadamente 0,5 $\mathrm{mg} / \mathrm{L}$ em águas com baixo teor de $\mathrm{Ca}^{2+}$ dissolvido (AZEVEDO e CHASIN, 2003). 
Tabela 1- Níveis de metais em amostras de água do lençol freático coletada em 2004, à montante e à jusante do Aterro Sanitário de Ribeirão Preto, S.P

Table 1- Metal level in groundwater samples, collected in 2004, upstream and downstream of the Ribeirão Preto Sanitary Landfill, Brazil

\begin{tabular}{|c|c|c|c|c|c|c|}
\hline Metal & Poço & Média & D. P. & Mínimo & Máximo & Mediana \\
\hline Ag & $\begin{array}{c}\text { Montante } \\
\text { Jusante }\end{array}$ & $\begin{array}{l}-- \\
---\end{array}$ & $\begin{array}{l}-- \\
---\end{array}$ & $\begin{array}{l}<0,002 \\
<0,002\end{array}$ & $\begin{array}{l}<0,002 \\
<0,002\end{array}$ & $\begin{array}{l}--- \\
---\end{array}$ \\
\hline Al & $\begin{array}{c}\text { Montante } \\
\text { Jusante }\end{array}$ & $\begin{array}{l}0,174 \\
0,013\end{array}$ & $\begin{array}{l}0,056 \\
0,002\end{array}$ & $\begin{array}{l}0,118 \\
0,009\end{array}$ & $\begin{array}{l}\mathbf{0 , 2 7 2} \\
0,018\end{array}$ & $\begin{array}{l}0,717 \\
0,013\end{array}$ \\
\hline $\mathbf{B a}$ & $\begin{array}{c}\text { Montante } \\
\text { Jusante }\end{array}$ & $\begin{array}{l}0,039 \\
0,083\end{array}$ & $\begin{array}{l}0,013 \\
0,044\end{array}$ & $\begin{array}{l}0,021 \\
0,046\end{array}$ & $\begin{array}{l}0,061 \\
0,164\end{array}$ & $\begin{array}{l}0,037 \\
0,068\end{array}$ \\
\hline $\mathbf{C a}$ & $\begin{array}{c}\text { Montante } \\
\text { Jusante }\end{array}$ & $\begin{array}{l}3,04 \\
5,58\end{array}$ & $\begin{array}{l}1,74 \\
1,12\end{array}$ & $\begin{array}{l}1,20 \\
2,89\end{array}$ & $\begin{array}{l}5,56 \\
7,40\end{array}$ & $\begin{array}{l}2,91 \\
5,68\end{array}$ \\
\hline Cd & $\begin{array}{c}\text { Montante } \\
\text { Jusante }\end{array}$ & $\begin{array}{l}--- \\
--- \\
\end{array}$ & $\begin{array}{l}--- \\
--- \\
\end{array}$ & $\begin{array}{l}<0,0001 \\
<0,0001\end{array}$ & $\begin{array}{l}<0,0001 \\
<0,0001\end{array}$ & $\begin{array}{l}--- \\
--- \\
\end{array}$ \\
\hline $\mathrm{Cr}$ & $\begin{array}{c}\text { Montante } \\
\text { Jusante }\end{array}$ & $\begin{array}{l}--- \\
---\end{array}$ & $\begin{array}{l}-- \\
---\end{array}$ & $\begin{array}{l}<0,002 \\
<0,002\end{array}$ & $\begin{array}{l}<0,002 \\
<0,002\end{array}$ & $\begin{array}{l}--- \\
---\end{array}$ \\
\hline $\mathbf{C u}$ & $\begin{array}{c}\text { Montante } \\
\text { Jusante }\end{array}$ & $\begin{array}{l}--- \\
---\end{array}$ & $\begin{array}{l}-- \\
---\end{array}$ & $\begin{array}{l}<0,005 \\
<0,005\end{array}$ & $\begin{array}{c}<0,005 \\
0,009\end{array}$ & $\begin{array}{l}--- \\
---\end{array}$ \\
\hline $\mathrm{Hg}$ & $\begin{array}{c}\text { Montante } \\
\text { Jusante }\end{array}$ & $\begin{array}{l}-- \\
---\end{array}$ & $\begin{array}{l}--- \\
---\end{array}$ & $\begin{array}{l}<0,0001 \\
<0,0001\end{array}$ & $\begin{array}{l}0,00030 \\
0,00032\end{array}$ & $\begin{array}{l}-- \\
---\end{array}$ \\
\hline $\mathbf{K}$ & $\begin{array}{c}\text { Montante } \\
\text { Jusante }\end{array}$ & $\begin{array}{l}--- \\
---\end{array}$ & $\begin{array}{l}--- \\
---\end{array}$ & $\begin{array}{l}<1,0 \\
<1,0\end{array}$ & $\begin{array}{l}1,529 \\
2,552\end{array}$ & $\begin{array}{l}--- \\
---\end{array}$ \\
\hline Mg & $\begin{array}{c}\text { Montante } \\
\text { Jusante }\end{array}$ & $\begin{array}{l}-- \\
3,16\end{array}$ & $\begin{array}{c}-- \\
0,41\end{array}$ & $\begin{array}{l}<2,0 \\
2,59\end{array}$ & $\begin{array}{l}<2,0 \\
3,75\end{array}$ & $\begin{array}{l}--- \\
3,17\end{array}$ \\
\hline Mn & $\begin{array}{c}\text { Montante } \\
\text { Jusante }\end{array}$ & $\begin{array}{l}\mathbf{0 , 1 4 3} \\
\mathbf{0 , 0 9 6}\end{array}$ & $\begin{array}{l}0,143 \\
0,091\end{array}$ & $\begin{array}{l}0,012 \\
0,002\end{array}$ & $\begin{array}{l}0,294 \\
0,189\end{array}$ & $\begin{array}{l}0,136 \\
0,098\end{array}$ \\
\hline $\mathbf{N a}$ & $\begin{array}{c}\text { Montante } \\
\text { Jusante }\end{array}$ & $\begin{array}{c}-- \\
19,290\end{array}$ & $\begin{array}{c}--- \\
27,683\end{array}$ & $\begin{array}{l}<<1,0 \\
1,194\end{array}$ & $\begin{array}{c}6,677 \\
73,970\end{array}$ & $\begin{array}{c}--- \\
5,678\end{array}$ \\
\hline $\mathbf{P b}$ & $\begin{array}{c}\text { Montante } \\
\text { Jusante }\end{array}$ & $\begin{array}{l}0,1868 \\
0,0453 \\
\end{array}$ & $\begin{array}{l}0,1717 \\
0,0365\end{array}$ & $\begin{array}{l}0,0247 \\
0,0075\end{array}$ & $\begin{array}{l}0,3960 \\
0,1296 \\
\end{array}$ & $\begin{array}{l}0,1357 \\
0,0383\end{array}$ \\
\hline Se & $\begin{array}{c}\text { Montante } \\
\text { Jusante }\end{array}$ & $\begin{array}{l}-- \\
--- \\
\end{array}$ & $\begin{array}{l}--- \\
--- \\
\end{array}$ & $\begin{array}{l}<0,008 \\
<0,008\end{array}$ & $\begin{array}{c}\mathbf{0 , 0 1 5} \\
<0,008\end{array}$ & $\begin{array}{l}--- \\
--- \\
\end{array}$ \\
\hline $\mathbf{Z n}$ & $\begin{array}{c}\text { Montante } \\
\text { Jusante }\end{array}$ & $\begin{array}{l}0,0191 \\
0,0305\end{array}$ & $\begin{array}{l}0,0035 \\
0,0146\end{array}$ & $\begin{array}{l}0,0151 \\
0,0156\end{array}$ & $\begin{array}{l}0,0240 \\
0,0527\end{array}$ & $\begin{array}{l}0,0189 \\
0,0250\end{array}$ \\
\hline
\end{tabular}

D.P: Desvio Padrão

A presença desse metal provoca anemia, dores de cabeça, fadiga, perda de peso, disfunção cognitiva e coordenação motora reduzida, além de possuir alto potencial carcinogênico (PASCALICCHIO, 2002). $\mathrm{O} \mathrm{Pb}^{\circ}$ é reconhecido como um dos mais nocivos metais, a maior parte do $\mathrm{Pb}^{\circ}$ é incorporada ao tecido ósseo, devido à semelhança entre as propriedades dos compostos de $\mathrm{Pb}^{\circ}$ e $\mathrm{Ca}^{2+}$, ou incorporado na corrente sanguínea, podendo inibir enzimas responsáveis pela catalisação de uma etapa essencial da hemoglobina, levando a episódios de anemia crônica (SEGURA- MUÑOZ et al., 2003).

Segundo estudo realizado em 2000, para avaliação de metais pesados na água do lençol freático na área de influência do ASRP, todos os resultados obtidos para os metais $\mathrm{Cd}^{2+}, \mathrm{Cr}^{\circ}, \mathrm{Cu}^{\circ}$, $\mathrm{Hg}^{\circ}, \mathrm{Mn}^{\circ}, \mathrm{Pb}^{\circ}$ e $\mathrm{Zn}^{2+}$ (Tab. 2), apresentaram-se inferiores aos valores considerados como nível de intervenção pela Portaria 1.469/2000 do Ministério da Saúde (BRASIL, 2000). Neste estudo, apesar da detecção de alguns metais em concentrações superiores às recomendadas pelas normas legais para solo e chorume, ainda não havia contaminação da água do lençol freático no local. Dentre os metais analisados na água do lençol freático $\mathrm{Mn}^{\circ}$ e $\mathrm{Pb}^{\circ}$ apresentaram-se acima dos níveis de detecção, com valores que variaram de 0,008 a $0,074 \mathrm{mg} / \mathrm{L}$ e 0,1 a $0,003 \mathrm{mg} / \mathrm{L}$ respectivamente (SEGURA-MUÑOZ, 2002). 
Tabela 2- Níveis de metais pesados detectados nas amostras de água coletada nos poços à jusante, no ano de 2000,na área do Aterro Sanitário de Ribeirão Preto, S.P

Table 2- Heavy metal levels in groundwater samples collected in dowstream points in 2000, in the Ribeirão Preto Sanitary Landfill, Brazil

\begin{tabular}{|c|c|c|c|}
\hline Metal Ponto de Coleta & Poço A & Poço B & Poço C \\
\hline Cádmio & $0,149 \pm 0,007$ & $0,147 \pm 0,011$ & $<0,1$ \\
\hline Mercúrio & $<0,1$ & $<0,1$ & $<0,1$ \\
\hline Cromo & $1,062 \pm 0,014$ & $0,698 \pm 0,013$ & $0,727 \pm 0,040$ \\
\hline Manganês & $22,080 \pm 1,090$ & $74,381 \pm 16,631$ & $8,880 \pm 0,429$ \\
\hline Chumbo & $3,063 \pm 0,267$ & $3,781 \pm 0,212$ & $<0,1$ \\
\hline Zinco & $84,456 \pm 1,076$ & $154,135 \pm 19,610$ & $7,875 \pm 0,645$ \\
\hline Cobre & $9,203 \pm 2,847$ & $20,905 \pm 0,440$ & $1,395 \pm 0,098$ \\
\hline
\end{tabular}

(Fonte: Segura-Muñoz, 2002)

Os resultados obtidos no presente estudo, demonstram que as atividades desenvolvidas no ASRP estão afetando as concentrações de metais na água do lençol freático no local, já que os poços à montante apresentaram, alterações de $\mathrm{Al}^{3+}, \mathrm{Mn}^{\circ}, \mathrm{Pb}^{\circ}$ e $\mathrm{Se}^{2-}$ e os poços à jusante do ASRP apresentaram alterações de $\mathrm{Mn}^{\circ}$ e $\mathrm{Pb}^{\circ}$; comparando-se tais resultados com os obtidos em estudo realizado no ano 2000 que não apresentou níveis de metais acima do estabelecido pela Portaria vigente nessa data, reafirma-se a necessidade de um constante monitoramento das águas do lençol freático a fim de minimizar ou impedir qualquer impacto ambiental concernente à contaminação por metais no local. Cabe destacar a periculosidade desses metais na saúde humana quando ultrapassam os limites estipulados pela Portaria 518/2004 do MS.

No ASRP, os poços à montante são de difícil contaminação por percolação do chorume devido à maior altitude, portanto, infere-se que a maior concentração de metais em tais poços pode estar diretamente relacionada à concentração mais ácida de $\mathrm{pH}$. Por este motivo salienta-se a importância da avaliação das condições de construção dos poços para verificar o motivo da detecção dos metais $\mathrm{Al}^{3+}, \mathrm{Mn}^{\circ}, \mathrm{Pb}^{\circ}$ e $\mathrm{Se}^{2-}$ à montante e $\mathrm{Mn}^{\circ}$ e $\mathrm{Pb}^{\circ}$ à jusante acima dos níveis estabelecidos pela Portaria 518/2004 do MS, bem como aprofundar o conhecimento do perfil hidrogeológico da área de influência do ASRP, pois o perfil geográfico dos poços de monitoramento do ASRP, pode ser diferente do perfil hidrogeológico do lençol freático abaixo do mesmo, assim como a direção do fluxo das águas. Não foram encontradas informações acerca do perfil hidrogeológico da área do ASRP na literatura científica, que são fundamentais para o esclarecimento dos dados obtidos. 


\section{REFERÊNCIAS}

AGENDA 21 BRASILEIRA, 2000, Brasília. Comissão de Políticas de desenvolvimento sustentável e da agenda 21 nacional. Brasília, 2000. $30-45$ p.

ALMEIDA, F.F.M. DE; CARNEIRO, C.D.R. Água para o Mercosul no século XXI. Ciência Hoje, v. 24, n.143, p. $36-43,2001$.

ALVEZ, Z.C. Meio Ambiente por inteiro: Qualidade da água. Jornal da Secretaria Municipal do Meio Ambiente, 1995. Ano 1. Caderno 1, p. 1 - 7.

AMERICAN PUBLIC HEALTH ASSOCIATION. Standard Methods for the Examination of water and Wastewater. $20^{\circ}$ Edição. Washington: Editora Washington (DC), 1998. 1085 p.

ASSOCIAÇÃO BRASILEIRA DE NORMAS TÉCNICAS. Resíduos Sólidos: Classificação. NBR 10.004. Rio de Janeiro: Editora ABNT, 1987.

AZEVEDO, F.A.; CHASIN, A.A.M. Metais: gerenciamento da toxicidade. São Paulo: Atheneu, 2003.

BITTENCOURT, A.V.L.; ROSA FILHO, E.F.; HINDI, E.C.; FILHO, A.C.B. A influência dos basaltos e de misturas com águas de aqüíferos sotopostos nas águas do Sistema Aqüífero Serra-Geral na Bacia do Rio Piriqui, Paraná - Br. Revista Água Subterrânea, São Paulo, n. 17, p. 7 - 13, mai. 2003.

BRASIL. Ministério da Saúde. Estabelece os procedimentos e responsabilidades relativos ao controle e vigilância da qualidade da água para consumo humano e seu padrão de potabilidade. Portaria No. 1469/2000, 01 de janeiro de 2001. Diário Oficial da União, Brasília, jan. 2001.

BRASIL. Ministério da Saúde. Estabelece os procedimentos e responsabilidades relativos ao controle e vigência de qualidade da água para consumo humano e seu padrão de potabilidade. Portaria No. 518/2004, 13 de março de 2004. Diário Oficial da União, Brasília, mar. 2006. DATASUS. Disponível em:

<http://w3.datasus.gov.br/datasus/datasus.php>
FERREIRA, P.C.; PIAI, K. A.; GALVIS-ALONSO, O.Y.; TAKAYANAGUI, A.M.M; SEGURAMUÑOZ, S.I. Alumínio como fator de risco para a Doença de Alzheimer. Arquivos de NeuroPsiquiatria, São Paulo, v. 63, p. 35 e 103, 2005 apresentado em Ribeirão Preto, SP.

PHILIPPI JÚNIOR, A.; ALVES, A. C. Questões de Direito Ambiental. São Paulo: CEPA , 2004.

PASCALICCHIO, A.E. Contaminação por metais pesados. Saúde Pública e Medicina Ortomolecular, São Paulo: Annablume, 2002.

REBOUÇAS, A.C.; AMORE, L. O Sistema Aqüífero Guarani - SAG. Revista Águas Subterrâneas No. 16/Maio 2002. ABAS. Associação Brasileira de Águas Subterrâneas.

ROSA FILHO, E.F.; BITTENCOURT, A.V.L.; HINDI, E.C.; ARAVENA, R. Caracterização hidroquímica do Aqüífero Guarani na porção centronorte do Estado do Paraná, Brasil. Revista Águas Subterrâneas, v.19, n.1, p. 87-94, 2005.

SEGURA-MUÑOZ, S.I. Impacto Ambiental na área do Aterro Sanitário e Incinerador de Resíduos Sólidos de Ribeirão Preto: uma avaliação dos níveis de metais pesados. Ribeirão Preto. 2002. 150p. Tese (Doutorado). Programa de Enfermagem em Saúde Pública. Escola de Enfermagem de Ribeirão Preto, Universidade de São Paulo.

SEGURA-MUÑOZ, S.I; TAKAYANAGHI, A.M.M; LOPES, T.M; TREVILATO, T.M.B; HERING, S. Estudo do efeito neurotóxico da exposição ocupacional ao mercúrio, ao chumbo e ao manganês utilizando como ferramenta metodológica a Revisão Sistemática de Literatura. O mundo da Saúde, São Paulo, v. 27, n. 4, p. 48 out./ dez. 2003.

SÃO PAULO. Secretaria do Meio Ambiente do Estado de São (Brasil). Secretaria de Meio Ambiente, Saúde Pública e Proteção ao consumidor do Estado da Baviera (Alemanha). Sistemas de informação para o gerenciamento ambiental dos recursos hídricos subterrâneos na área de afloramento do Aquífero Guarani no Estado de São Paulo. 2004.

VIARTI, P. Água escassa. Revista Revida, n.11, p.13-16, 1999. 
Análise dos níveis de metais em água subterrânea coletada à montante e jusante do aterro sanitário de Ribeirão Preto, Brasil 